\title{
GYNECOMASTIA AND BREAST PAIN INDUCED BY ADJUVANT THERAPY WITH BICALUTAMIDE AFTER RADICAL PROSTATECTOMY IN PATIENTS WITH PROSTATE CANCER: THE ROLE OF TAMOXIFEN AND RADIOTHERAPY
}

\author{
GIUSEPPE DI LORENZO, SISTO PERDONÀ, SABINO DE PLACIDO, MASSIMO D'ARMIENTO, \\ ANTONIO GALLO, ROCCO DAMIANO, DOMENICO PINGITORE, LUIGI GALLO, MARCO DE SIO \\ AND RICCARDO AUTORINO*
}

From the Dipartimento di Endocrinologia e Oncologia Molecolare e Clinica, Università degli Studi Federico II (GDL, SDP), Operating Unit Urologia, Istituto Nazionale Tumori, Fondazione "G. Pascale" Istituto Nazionale Ricerca sul Cancro Napoli (SP, AG, LG) and

Clinica Urologica, Seconda Università degli Studi (MD’A, MDS, RA), Napoli and Cattedra di Urologia, Università degli Studi Magna Graecia (RD) and Operating Unit Radioterapia, Ospedale Ciaccio (DP), Catanzaro, Italy

\section{ABSTRACT}

Purpose: We investigated the role of tamoxifen and radiotherapy (RT) for the prevention and treatment of gynecomastia and breast pain during adjuvant bicalutamide monotherapy after radical prostatectomy $(\mathrm{RP})$ in patients with prostate cancer. Also, we evaluated their effects on patient hormonal status, quality of life (QOL), sexual function and prostate specific antigen relapse-free survival.

Materials and Methods: This was a multicenter prospective trial. From January 2002 to February 2004, 102 patients who had undergone RP for localized or locally advanced prostate cancer were recruited and randomized into 3 groups, namely group 1-those receiving only 150 mg bicalutamide as adjuvant hormonal therapy, group 2-those receiving bicalutamide and 10 mg tamoxifen, and group 3-those receiving bicalutamide and RT. Patients in group 1 in whom gynecomastia or breast pain developed were subsequently randomized to receive tamoxifen or RT soon after symptoms started. Gynecomastia, breast pain, prostate specific antigen, QOL, sexual function and hormonal levels were assessed. Minimum followup was 12 months.

Results: Of group 1 patients $67 \%$ had gynecomastia compared with $8 \%$ in group 2 and $34 \%$ in group 3. Breast pain was more frequent in group 1 than in groups 2 and 3 (58\% vs 7\% and 30\%, respectively). Differences were significant between groups 1 and 2 (OR $0.12 \mathrm{p}<0.001$ ), and groups 1 and 3 (OR $0.52 \mathrm{p}<0.01$ ). In patients in group 1 who had gynecomastia or breast pain a significant decrease in symptoms was achieved in those receiving tamoxifen $(p<0.05)$. Treatments were well tolerated in the 3 groups. No differences in QOL between groups 2 and 3 were found. At a median followup of 26 months we observed 12 biochemical relapses.

Conclusions: Gynecomastia and breast pain induced by bicalutamide monotherapy after RP can be prevented and treated. Tamoxifen has been shown to be more effective and safe than RT in this setting. QOL and sexual function are not negatively influenced by these 2 treatment options.

KEY WORDS: prostate, prostatectomy, quality of life, gynecomastia, pain

A treatment option in patients with early prostate cancer $(\mathrm{PCa})$ is radical prostatectomy $(\mathrm{RP})$ and most men undergoing this surgical treatment have an excellent outcome. ${ }^{1}$ However, a significant proportion of patients can experience disease recurrence and prostate specific antigen (PSA) progression is the earliest evidence of persistent or recurrent disease after primary therapy with curative intent. ${ }^{2}$

In previously untreated patients with nonmetastatic $\mathrm{PCa}$ $150 \mathrm{mg}$ bicalutamide monotherapy has been shown to be equivalent to castration in terms of the survival rate at a median followup of 6.3 years, offering quality of life (QOL) advantages with respect to sexual interest and physical capacity. $^{3,4}$ The value of adding $150 \mathrm{mg}$ bicalutamide daily to standard care for early $\mathrm{PCa}$ is being investigated in the

Submited for publication March 24, 2005.

Study received approval from the ethics committee at each participating center.

* Correspondence: Vico Santo Spirito 54, 80132 Napoli, Italy (telephone and FAX: + 39 08119568212; e-mail: giuseppedilorenzoncol@ hotmail.com). bicalutamide early prostate cancer (EPC) program, which is the largest international clinical trial of early $\mathrm{PCa}$ therapy to date. $^{5,6}$ The EPC program is ongoing and data on the effect of treatment on mortality are still missing. Followup will provide further clarification on the role of bicalutamide in this setting.

To date $150 \mathrm{mg}$ bicalutamide monotherapy has not been Food and Drug Administration approved for use in the United States. Nonetheless, it has been licensed in some European countries as adjuvant treatment for early PC. Notably in Italy based on EPC program toxicity data $150 \mathrm{mg}$ bicalutamide should be prescribed with caution as adjuvant treatment in patients at low risk who have not received any primary treatment with curative intent

In the EPC program the incidence of gynecomastia and breast pain is $68.3 \%$ and $73.6 \%$, respectively, with symptoms developing in the majority of patients within the first 6 to 9 months of bicalutamide therapy. ${ }^{6}$ Controlled trials have demonstrated the efficacy of prophylactic estrogen therapy 
and prophylactic breast irradiation for gynecomastia and breast pain caused by bicalutamide monotherapy. ${ }^{7,8}$

We investigated the role of $10 \mathrm{mg}$ tamoxifen daily and 12 Gy electron beam radiotherapy (RT) for preventing and treating gynecomastia and breast pain during $150 \mathrm{mg}$ bicalutamide monotherapy in adjuvant setting after RP. The effects of these 2 treatment options on patient hormonal status, QOL, sexual function and prostate specific antigen (PSA) relapse-free survival were also evaluated.

\section{MATERIALS AND METHODS}

Patient recruitment. This multicenter, randomized trial was done between January 2002 and February 2004. The study population consisted of men with histologically confirmed prostate cancer without distant metastases (T1-T3, any N, M0) and no evidence of current gynecomastia or breast pain. All patients had received RP with or without a nerve sparing (NS) procedure as primary therapy.

Exclusion criteria were previous hormonal therapy for $\mathrm{PCa}$, metastatic disease, evidence of biochemical relapse after RP, any comorbid condition that could contraindicate trial drugs, or hematological (hemoglobin $10 \mathrm{gm} / \mathrm{dl}$ or less, white blood count less than $3,000 / \mu \mathrm{l}$ and platelet count less than $100,000 / \mu \mathrm{l}$ ), renal (creatinine $2.2 \mathrm{ng} / \mathrm{nl}$ or greater) or liver (transaminase and bilirubin 50\% of normal or greater) dysfunction.

The trial was performed in accordance with the 1964 Declaration of Helsinki. Each patient provided written informed consent. The protocol was approved by the ethics committee at each participating center.

Study design. Treatment was assigned on a randomized basis according to a 1:1:1 ratio. Randomization was performed centrally at Pascale Cancer Institute of Naples using a stratified, permuted randomization algorithm that was balanced within institutions. Stratification factors were disease stage (T1-T2 vs T3), NS procedure (yes vs no), lymph node involvement ( $\mathrm{N}+$ vs $\mathrm{N}-$ vs $\mathrm{NX}$ ), Gleason score (less than 7 vs 7 or greater) and PSA (less than $10 \mathrm{vs} 10 \mathrm{ng} / \mathrm{ml}$ or greater). All randomly assigned patients were included in efficacy and safety analyses.
All patients were divided into 3 groups (fig. 1). Group 1 patients received only $150 \mathrm{mg}$ bicalutamide daily. Those presenting a gynecomastia score of 3-4 or moderate-severe breast pain were successively randomized to receive $10 \mathrm{mg}$ tamoxifen daily for 24 weeks or a single 12 Gy dose of RT. Group 2 patients were treated with $150 \mathrm{mg}$ bicalutamide daily and $10 \mathrm{mg}$ tamoxifen daily for 24 weeks. Group 3 patients were treated with $150 \mathrm{mg}$ bicalutamide daily and a single 12 Gy dose of RT. RT was administered as an electron beam directed to irradiate a $5 \mathrm{~cm}$ diameter circle of tissue centered around each nipple.

Oncological followup. Physical examination, hematology and serum biochemistry evaluations, including total PSA, were performed every 3 months. Radiological assessments, ie computerized tomography, bone scan, abdominal ultrasonography and chest x-ray, were performed when disease progression was suspected based on PSA. PSA progression was defined as 2 consecutive PSA increases (greater than $0.04 \mathrm{ng} /$ $\mathrm{ml}$ ).

Gynecomastia / breast pain assessment. Calipers were used to measure gynecomastia. The severity of gynecomastia was scored based on the largest diameter, including grade $1-2$ or less, grade 2 -between 2 and 4 , grade 3 -between 4 and 6 , and grade $4-$ greater than $6 \mathrm{~cm}$. Breast pain was evaluated via direct patient questioning at each visit. It was arbitrarily scored according to severity as none, mild, moderate or severe. Gynecomastia and breast pain were evaluated monthly.

QOL assessment. QOL was evaluated using the European Organisation for Research and Treatment of Cancer (EORTC) QLQ-C30 questionnaire, which was developed by the EORTC to measure QOL in patients with cancer in clinical trials. ${ }^{9}$ The questionnaire was administered at baseline and at 3-month intervals during treatment.

Erectile function assessment. Erectile function was evaluated only in patients who had undergone NS RP using the abridged 5 item version of the International Index of Erectile Function (IIEF-5) questionnaire. ${ }^{10}$ The study population consisted only of patients with normal erectile function (score 21 to 25) or mild erectile dysfunction (score 15 to 20), as assessed before surgery. In these patients rehabilitation therapy was

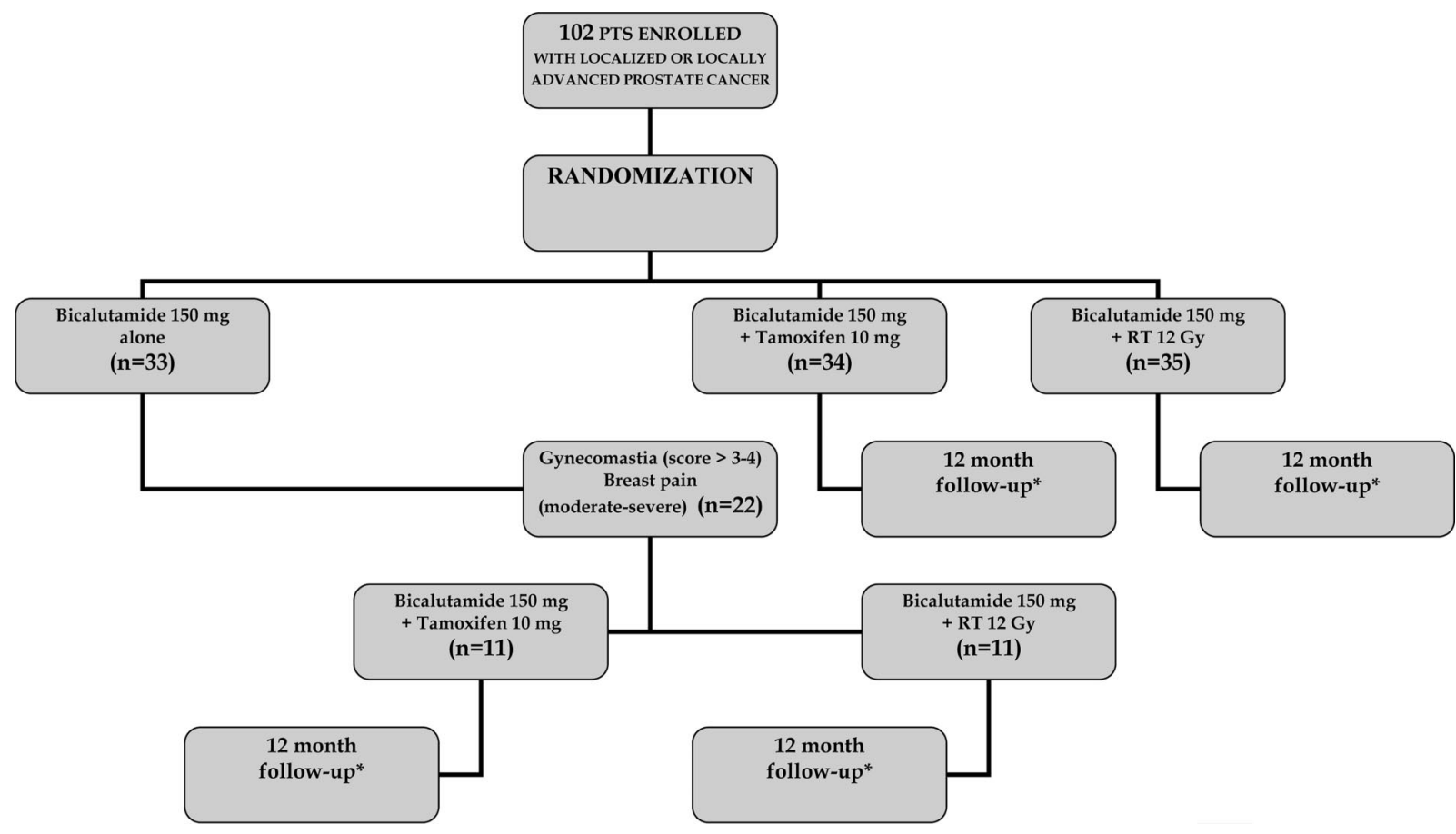

FIG. 1. Study design. Asterisk indicates monthly assessment with breast pain/gynecomastia evaluations, and 3-month assessment with PSA, laboratory examinations, QOL and IIEF-5 questionnaires. 
TABLE 1. Patient demographics

\begin{tabular}{|c|c|c|c|c|}
\hline & Group 1 & Group 2 & Group 3 & Totals \\
\hline No. pts & 33 & 34 & 35 & 102 \\
\hline \multicolumn{5}{|l|}{ Age: } \\
\hline Median & 65.5 & 67.5 & 67.5 & 66.8 \\
\hline Range & $50-75$ & $57-74$ & $50-73$ & $50-75$ \\
\hline \multicolumn{5}{|l|}{ No. stage: } \\
\hline T1-2 & 22 & 22 & 22 & 66 \\
\hline T3 & 11 & 12 & 13 & 36 \\
\hline \multicolumn{5}{|c|}{ No. Gleason score: } \\
\hline Less than 7 & 20 & 20 & 21 & 61 \\
\hline 7 or Greater & 13 & 14 & 14 & 41 \\
\hline \multicolumn{5}{|c|}{ No. PSA before RP $(\mathrm{ng} / \mathrm{ml})$ : } \\
\hline Less than 10 & 20 & 20 & 22 & 62 \\
\hline 10 or Greater & 13 & 14 & 13 & 40 \\
\hline \multicolumn{5}{|l|}{ No. node status: } \\
\hline $\mathrm{N}+$ & 1 & 2 & 2 & 5 \\
\hline $\mathrm{N}-$ & 25 & 26 & 26 & 77 \\
\hline $\mathrm{Nx}$ & 7 & 6 & 7 & 20 \\
\hline \multicolumn{5}{|l|}{ No. RP: } \\
\hline NS & 22 & 23 & 22 & 67 \\
\hline No NS & 11 & 11 & 13 & 35 \\
\hline
\end{tabular}

started at randomization using intracavernous injection with $10 \mu \mathrm{g}$ alprostadil twice weekly for month 1 and with type 5 phosphodiesterase inhibitors thereafter at the maximum dose available, ingested on demand. Patients were encouraged to have sexual activity. The IIEF-5 questionnaire was administered at baseline (at randomization) and at 3-month intervals during treatment.

Pharmacodynamic assessment. Blood samples were obtained at baseline, and 3 and 6 months, Testosterone, free testosterone and prolactin were assessed by radioimmunoassay.

Statistical methods. The chi-square and Fisher's exact tests were used to compare groups in respect to the incidence of gynecomastia, breast pain, adverse effects, QOL and IIEF-5 scores, and pharmacodynamics, showing the OR be- tween groups 1 and 2 , and 1 and 3. PSA relapse-free survival was estimated with the Kaplan-Meier product limit method. ${ }^{11}$ All $p$ values are 2 -sided and only $\mathrm{p} \leq 0.05$ is shown in the tables.

\section{RESULTS}

Overall 102 patients were randomized and included in the analysis. Patients and disease characteristics were well balanced among treatment groups (table 1). Minimum followup was 12 months (median 26, range 13 to 35 ) in all patients.

Efficacy. Figure 2 shows the incidence of grade 3-4 gynecomastia and moderate-severe breast pain after 6 months in each group. In group 1, 67\% of patients had gynecomastia compared with $8 \%$ in group 2 and $34 \%$ in group 3 . The difference was statistically significant between groups 1 and 2 (OR $0.12,95 \%$ CI 0.10 to $0.15, \mathrm{p}<0.001$ ), and groups 1 and 3 (OR $0.52,95 \%$ CI 0.49 to 0.54 , p <0.01). Breast pain was more frequent in group 1 than in groups 2 and $3(58 \%$ vs $7 \%$ and $30 \%$, respectively). Differences in breast pain were statistically significant between groups 1 and 2 (OR 0.13, $95 \%$ CI 0.10 to $0.16, \mathrm{p}<0.001$ ), and groups 1 and 3 (OR 0.44 , $95 \%$ CI 0.41 to $0.47, \mathrm{p}<0.025$ ).

Considering that $67 \%$ and $58 \%$ of group 1 patients had grade 3-4 gynecomastia and breast pain, respectively, 22 were successively randomized to receive tamoxifen (11) or RT (11). These patients included 19 with breast pain who were successively treated with tamoxifen (10) or RT (9).

A significant decrease in gynecomastia was achieved in patients receiving tamoxifen $(\mathrm{p}<0.05)$. In fact, after 6 and 9 months gynecomastia was reported in only 1 of 11 of these patients (9\%), while 6 of $11(54 \%)$ treated with RT still reported gynecomastia (fig. 3). Figure 4 shows breast pain relief results. A higher decrease was reported by patients in the tamoxifen group than in the RT group $(\mathrm{p}<0.05)$. In fact, after 6 and 9 months breast pain were reported in only 2 of 10

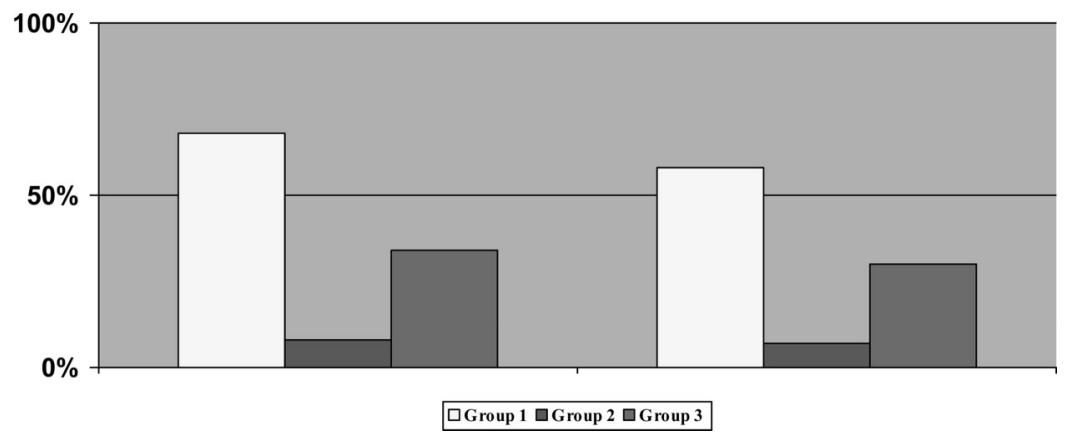

FIG. 2. Incidence of grade 3-4 gynecomastia (left) and moderate-severe breast pain (right) in 3 groups after 6 months. Differences in gynecomastia incidence were significant between groups 1 and 2 (OR $0.12,95 \%$ CI 0.10 to $0.15, \mathrm{p}<0.001$ ), and groups 1 and 3 (OR 0.52 , 95\% CI 0.49 to $0.54, \mathrm{p}<0.01)$. Differences in breast pain were statistically significant between groups 1 and 2 (OR $0.13,95 \%$ CI 0.10 to 0.16 , $\mathrm{p}<0.001)$ and groups 1 and 3 (OR $0.44,95 \%$ CI 0.41 to $0.47, \mathrm{p}<0.025$ ).

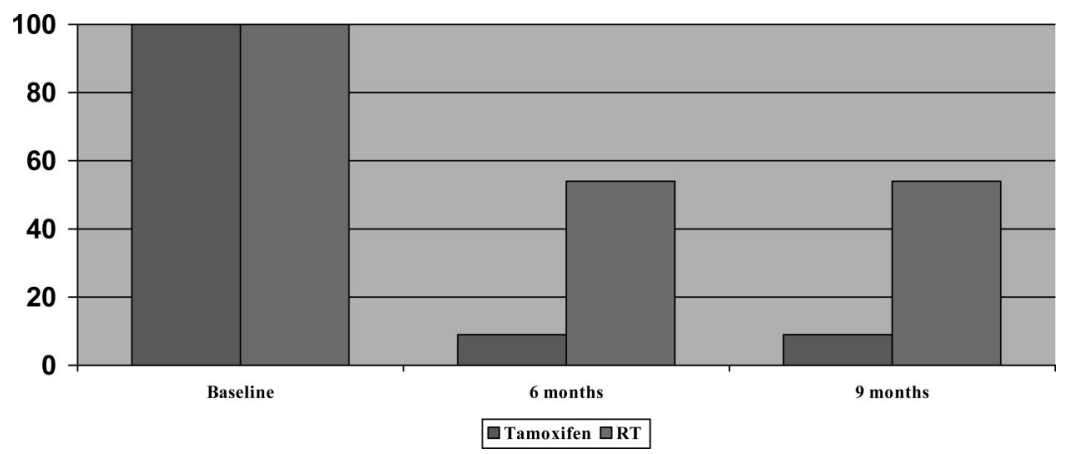

FIG. 3. Results of tamoxifen treatment or RT in 22 group 1 patients who had gynecomastia. Significant decrease in gynecomastia was noted in patients receiving tamoxifen $(\mathrm{p}<0.05)$. 


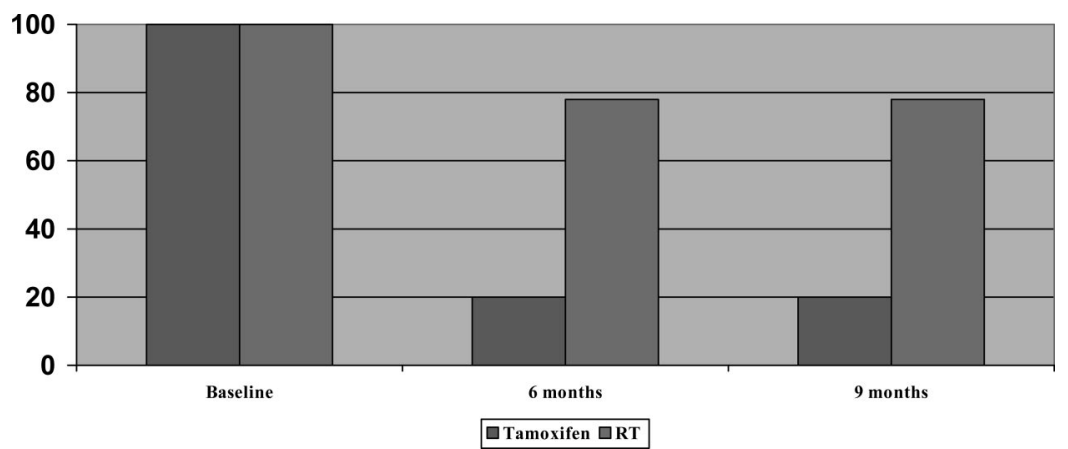

FIG. 4. Results of tamoxifen treatment or RT in 19 group 1 patients who had moderate-severe breast pain. Breast pain was more decreased in tamoxifen group $(\mathrm{p}<0.05)$.

\begin{tabular}{|c|c|c|c|}
\hline Side Effects & $\%$ Group 1 & \% Group 2 & $\%$ Group 3 \\
\hline No. pts & 33 & 34 & 35 \\
\hline Rash/nipple erythema* & 31 & 3 & 40 \\
\hline Skin irritation* & 0 & 0 & 40 \\
\hline Pruritis & 3 & 6 & 3 \\
\hline Anemia & 0 & 2 & 0 \\
\hline Fever & 0 & 2 & 2 \\
\hline Myelotoxicity & 2 & 0 & 4 \\
\hline Asthenia & 3 & 3 & 6 \\
\hline Cardiovascular events & 3 & 6 & 6 \\
\hline Neurological events & 3 & 3 & 3 \\
\hline Constipation & 6 & 9 & 6 \\
\hline Diarrhea & 6 & 6 & 6 \\
\hline Hot flashes & 6 & 6 & 6 \\
\hline
\end{tabular}

men $(20 \%)$ receiving tamoxifen, while 7 of $9(78 \%)$ treated with RT still reported breast pain.

Adverse events. Treatments were well tolerated in the 3 groups (table 2). All RT related adverse events resolved and were short-lived with a median duration of 4 weeks.

QOL. Figure 5 shows global health scores in the 2 groups. Table 3 lists functional and symptom scales only in the 2 prevention arms, ie groups 2 and 3. Those data showed no differences between the groups.

Erectile function. A total of 52 patients were included in the analysis of sexual function. Figure 6 lists IIEF-5 scores. We found an increase in mean IIEF-5 scores at 6 and 12 months in all 3 groups without any significant differences among them.

Pharmacodynamics. Testosterone increased in all groups at 6 months without differences among the groups (fig. 7). Similarly free testosterone increased at the same time in patients in groups $1(\mathrm{p}<0.05)$ and $3(\mathrm{p}<0.05)$, while in group 2 patients it remained substantially unchanged (fig. 8). Prolactin remained unchanged in groups 1 and 3 , whereas there was a trend toward decreased levels in group $2(p<0.065$, fig. 9).

PSA relapse. Overall $88 \%$ of the patients were disease-free at a median followup of 26 months (range 12 to35). Figure 10 shows PSA relapse-free survival according to the KaplanMeier estimate in the study population. It seems that there were no differences among the 3 groups since there were 4 PSA relapses in each of groups 1 to 3 .

\section{DISCUSSION}

Recently the large international EPC program indicated the progression-free survival benefits of immediate $150 \mathrm{mg}$ bicalutamide treatment given in addition to standard care (RP, RT or watchful waiting) compared with standard care alone in patients with locally advanced PCa. ${ }^{6}$ Gynecomastia and breast pain are commonly reported adverse events of bicalutamide monotherapy and they may cause some patients to withdraw from treatment. Several interventions have been used as prevention, including surgery, hormone therapy and radiation..$^{7,8}$

Data from old studies of patients with $\mathrm{PCa}$ who were treated with the estrogen antagonist tamoxifen ${ }^{7}$ are today supported by recently published trials confirming that hormonal treatment can be used to restore the balance of estrogen and androgen. ${ }^{12,13}$ Boccardo $^{12}$ and Saltzstein ${ }^{13}$ et al recently reported 2 randomized trials of the role of tamoxifen and anastrozole for the prevention of gynecomastia and breast pain.

Studies of prophylactic breast irradiation before estrogen or antiandrogen therapy have shown success in a high pro-

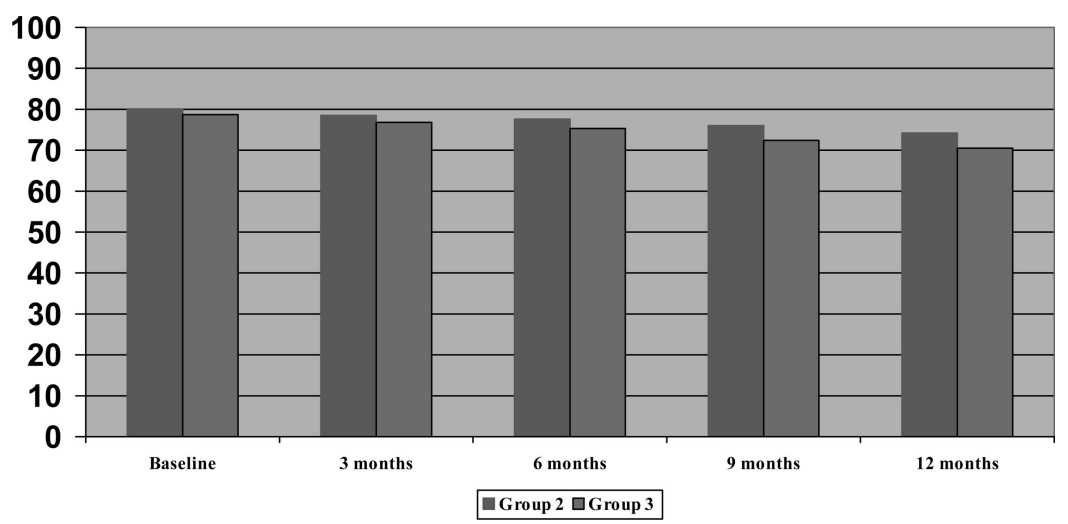

FIG. 5. Mean EORTC QLQ-C30 global health scores in 2 prevention arms (data presented as mean value). Score range was 1-very poor to 7 - excellent. For analysis raw questionnaire scores were transformed into 100 point scale. Calculated scores were 0 to 100 with higher scores representing higher level of functioning. 
TABLE 3. EORTC QLQ-C30 functional and symptom scales in 2 prevention groups

\begin{tabular}{|c|c|c|c|c|c|c|c|c|c|c|}
\hline \multirow{2}{*}{ Item } & \multicolumn{5}{|c|}{ Mean Group 2} & \multicolumn{5}{|c|}{ Mean Group 3} \\
\hline & Baseline & 3 Mos & $6 \mathrm{Mos}$ & $9 \mathrm{Mos}$ & $12 \mathrm{Mos}$ & Baseline & 3 Mos & $6 \mathrm{Mos}$ & 9 Mos & $12 \mathrm{Mos}$ \\
\hline \multicolumn{11}{|l|}{ Functional scales: } \\
\hline Physical & 84.2 & 80.2 & 79.2 & 78 & 77.4 & 83.8 & 82.2 & 81.1 & 78.3 & 77.6 \\
\hline Role & 77.4 & 77.2 & 76.5 & 76.7 & 76.1 & 76.9 & 76.4 & 75.8 & 76.5 & 74.6 \\
\hline Emotional & 79.4 & 80.0 & 80.5 & 80.7 & 81.3 & 79.4 & 79.6 & 79.6 & 78.3 & 79.7 \\
\hline Cognitive & 88.1 & 88.9 & 90.3 & 90.1 & 89.8 & 88.9 & 88.0 & 89.8 & 88.7 & 88.7 \\
\hline Social & 76.6 & 76.0 & 76.5 & 76.3 & 76.0 & 76.2 & 75.9 & 75.5 & 75.2 & 75.3 \\
\hline \multicolumn{11}{|l|}{ Symptom scales: } \\
\hline Fatigue & 25.5 & 23.8 & 22.6 & 22.6 & 23.4 & 25.2 & 24.6 & 23.8 & 24.2 & 24.1 \\
\hline Pain & 18.2 & 17.4 & 18.7 & 19.1 & 21.4 & 18.0 & 17.3 & 18.8 & 20.2 & 22.5 \\
\hline Sleep disturbance & 24.4 & 24.0 & 25.4 & 25.7 & 26.0 & 24.2 & 24.3 & 25.9 & 26.8 & 26.6 \\
\hline Constipation & 22.6 & 21.8 & 21.6 & 22.0 & 22.8 & 23.1 & 22.3 & 22.8 & 23.0 & 23.6 \\
\hline Diarrhea & 20.6 & 21.1 & 21.6 & 22.5 & 22.4 & 21.0 & 21.4 & 22.1 & 22.0 & 22.3 \\
\hline
\end{tabular}

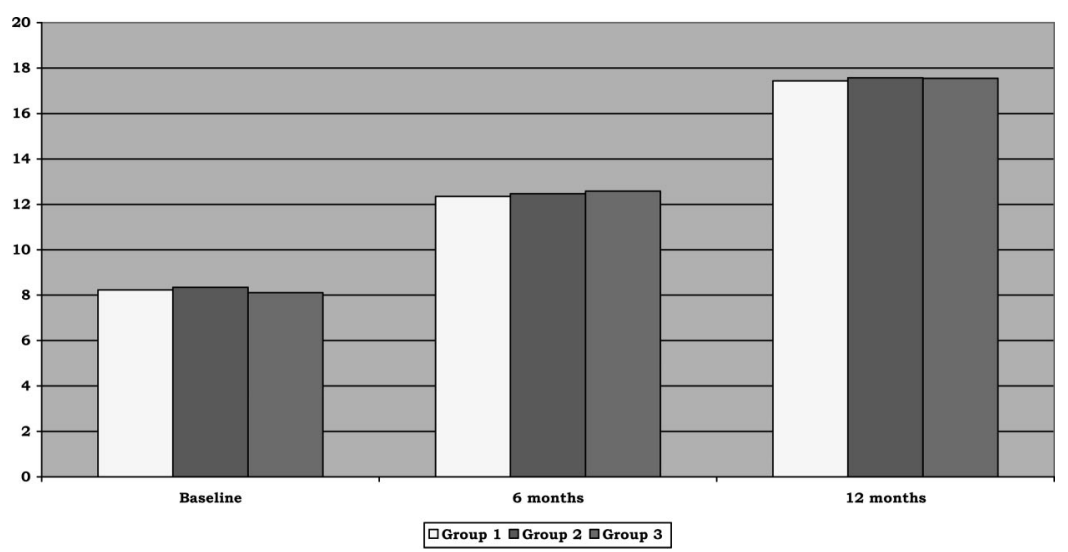

FIG. 6. Mean IIEF-5 scores in 52 evaluable patients. No significant differences were found between groups

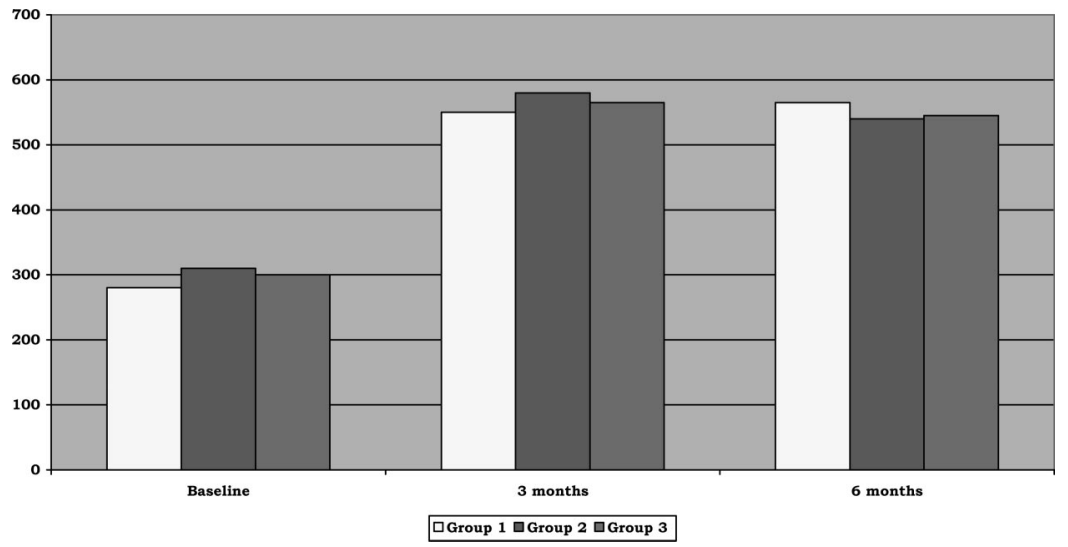

FIG. 7. Mean testosterone in ng/dl in 3 groups. No significant differences were found between groups

portion of patients. ${ }^{8}$ Tyrrell et al recently observed that prophylactic breast irradiation is an effective and well tolerated strategy for preventing bicalutamide induced gynecomastia. ${ }^{14}$ In this trial a single 10 Gy dose of electron beam RT significantly decreased the incidence of gynecomastia to $52 \%$ of patients. Van Poppel et al evaluated the efficacy of localized RT of 2, $6 \mathrm{~Gy}$ fractions in 51 patients receiving $150 \mathrm{mg}$ bicalutamide. ${ }^{15}$ Therapeutic RT improved the intensity of bicalutamide induced gynecomastia and/or breast pain in approximately a third of patients.

To our knowledge no randomized trials comparing RT and hormone therapy as treatment and prevention options for bicalutamide induced gynecomastia and breast pain have been reported to date. We performed such a comparative study to investigate the efficacy of tamoxifen and RT for gynecomastia and breast pain due to bicalutamide mono- therapy. The incidence of gynecomastia in the current study was similar to that in the EPC program but lower than that in the study of Boccardo et al $(67 \%, 68.3 \%$ and $73 \%$, respectively). ${ }^{12}$

Tamoxifen was more effective than RT for preventing bicalutamide induced gynecomastia and breast pain. Our positive findings are in agreement with prior studies with different treatment schedules, such as those of Boccardo ${ }^{12}$ and Saltzstein ${ }^{13}$ et al, who administered $20 \mathrm{mg}$ tamoxifen daily, and those of Eaton et al, who administered $20 \mathrm{mg}$ weekly. ${ }^{16}$

The incidence of gynecomastia in our trial was $34 \%$ after $\mathrm{RT}$, lower than that reported by Tyrrell et al, who noted gynecomastia in $52 \%$ of the RT group, ${ }^{14}$ and similar to that reported by Widmark et al, who observed gynecomastia in $28 \%$ of irradiated patients. ${ }^{17}$ These differences in the results can be probably attributed to the difference in the radiation 


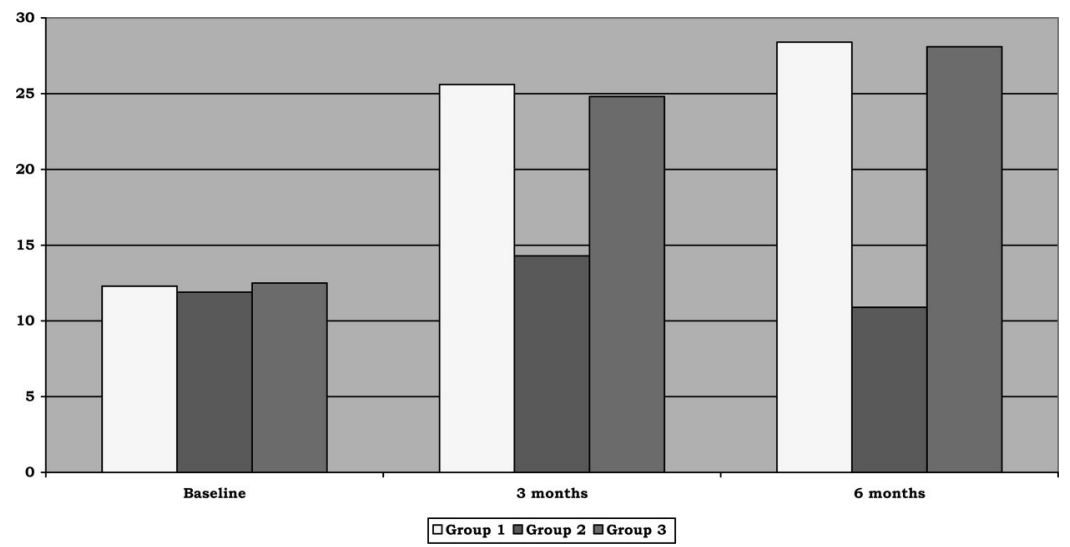

Fig. 8. Mean free testosterone in $\mathrm{pg} / \mathrm{ml}$ in 3 groups. Level was increased in groups 1 and 3 (each p <0.05), while in group 2 it was substantially unchanged.

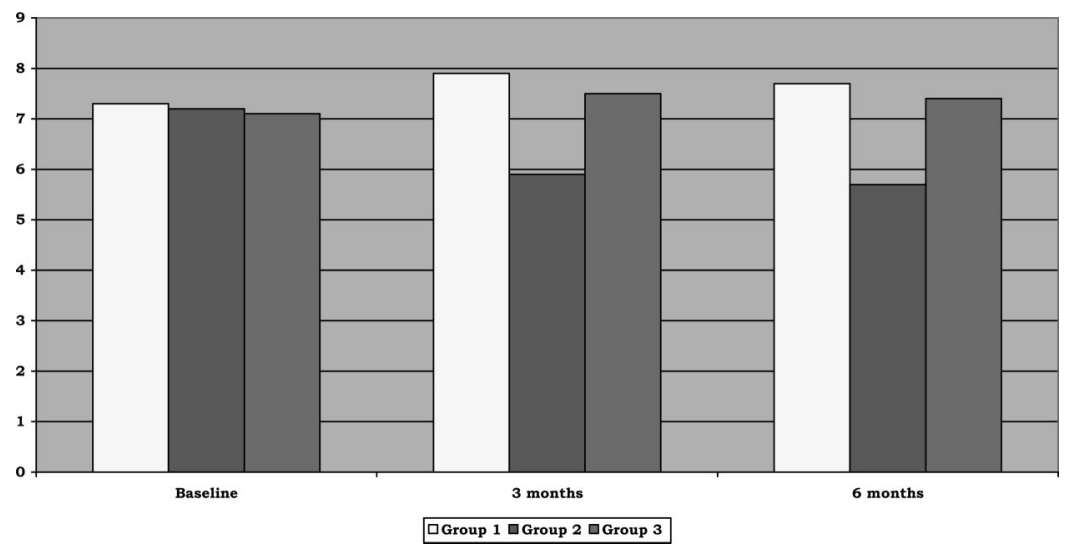

FIG. 9. Mean prolactin in $\mathrm{ng} / \mathrm{ml}$ in 3 groups. Levels remained unchanged in groups 1 and 3, whereas there was trend toward decreased levels in group $2(\mathrm{p}<0.065)$.

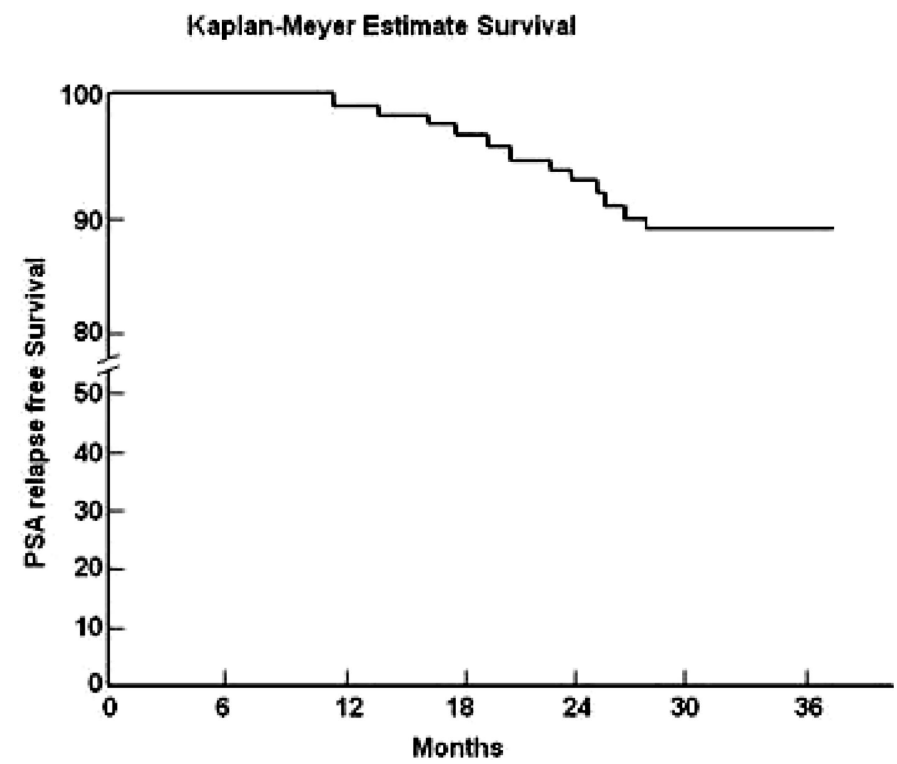

FIG. 10. PSA relapse-free survival according to Kaplan-Meier estimate in study population.

dose. We are aware that this is only an assumption. However, we did not have the possibility of performing a comparative study evaluating different radiation dosing or schedules. Moreover, to our knowledge there are no such comparative studies in the literature.

Considering that 22 of 33 patients (67\%) in group 1 had grade 3-4 gynecomastia, we further randomized them to receive tamoxifen or radiotherapy. Results were better with tamoxifen.

Our result is better than that reported by Van Poppel et al, who observed improvement in gynecomastia in a third of their patients. ${ }^{15}$ This difference may have been due to the difference in the number of RT fractions ( 12 Gy as a single fraction vs 2 fractions of 6 Gy each). ${ }^{18}$

RT related adverse events were seen in $40 \%$ of 35 patients and they were similar to those observed in previous studies, ${ }^{14,15}$ All were transient and resolved spontaneously. When designing this trial, the aim was to minimize the risk of adverse events and also minimize patient inconvenience by administering a single low dose of radiation. To date there have been no reports in the literature of secondary malignancy associated with a single 10 to 12 Gy dose. ${ }^{19}$

QOL evaluation and erectile function recovery after surgery were not negatively influenced by either treatment option.

The current study confirms that bicalutamide monotherapy causes an increase in testosterone and free testosterone. However, there were differences between groups in free testosterone at 6 months with significant increases in groups 1 and 3 ( $\mathrm{p}<0.05$ and $\mathrm{p}<0.05$ vs baseline, respectively) but not in the tamoxifen group. The maintenance of free testosterone in the tamoxifen group could suggest potential antitumor efficacy by antiestrogen.

The use of tamoxifen in prostate cancer is experimental and it is unclear what influence tamoxifen could have on progression and survival in these patients. For this question this supposed antitumor efficacy currently needs confirma- 
tion. In this respect after 26 months of median followup only 12 relapses were observed. It is reassuring that no differences in PSA relapse were recorded among the 3 groups.

\section{CONCLUSIONS}

Gynecomastia and breast pain induced by bicalutamide monotherapy after RP can be prevented and treated. Tamoxifen has shown to be more effective than RT in this setting without increasing adverse events and without compromising QOL, erection recovery after surgery and PSA relapsefree survival. However, the results of our study are preliminary and they should be considered with caution. More appropriate trials are needed to investigate the safety and efficacy of tamoxifen in combination with bicalutamide in patients with PCa.

\section{REFERENCES}

1. Amling, C. L., Blute, M. L., Bergstralh, E. J., Seay, T. M., Slezak, J. and Zincke, H.: Long-term hazard of progression after radical prostatectomy for clinically localized prostate cancer: continued risk of biochemical failure after 5 years. J Urol, 164: 101,2000

2. Iversen, P., Tyrrell, C. J., Kaisary, A. V., Anderson, J. B., Van Poppel, H., Tammela, T. L. J. et al: Bicalutamide monotherapy compared with castration in patients with nonmetastatic locally advanced prostate cancer: 6.3 years of followup. J Urol, 164: 1579,2000

3. Boccardo, F., Rubagotti, A., Barichello, M., Battaglia, M., Carmignani, G., Comeri, G. et al: Bicalutamide monotherapy versus flutamide plus goserelin in prostate cancer patients: results of an Italian Prostate Cancer Project study. J Clin Oncol, 17: 2027, 1999

4. Chatelain, C., Fourcade, R. O. and Delchambre, J.: Bicalutamide versus combined androgen blockade: open French multicentre trial in patients with metastatic prostate cancer. Br J Urol, 80: 283, abstract 1111, 1997

5. See, W. A., Wirth, M. P., McLeod, D. G., Iverson, P., Klimberg, I., Gleason, D. et al: Bicalutamide as immediate therapy either alone or as adjuvant to standard care of patients with localized or locally advanced prostate cancer: first analysis of the early prostate cancer program. J Urol, 168: 429, 2002

6. Wirth, M. P., See, W. A., McLeod, D. G., Iversen, P., Morris, T. and Carroll, K.: Bicalutamide $150 \mathrm{mg}$ in addition to standard care in patients with localized or locally advanced prostate cancer: results from the second analysis of early prostate cancer program at median followup of 5.4 years. J Urol, 172: 1865, 2004

7. McLeod, D. G. and Iversen, P.: Gynecomastia in patients with prostate cancer: a review of treatment options. Urology, 56: 713,2000

8. Dicker, A. P.: The safety and tolerability of low-dose irradiation for the management of gynaecomastia caused by antiandrogen monotherapy. Lancet Oncol, 4: 30, 2003

9. Aaronson, N. K., Ahmedzai, S., Bergman, B., Bullinger, M., Cull, A., Duez, N. J. et al: The European Organization for Research and Treatment of Cancer QLQ-C30: a quality-of-life instrument for use in international clinical trials in oncology. J Natl Cancer Inst, 85: 365, 1993

10. Rosen, E. L., Teloken, C., Sogari, P. R. and Vargas Souto, C. A.: The use of the simplified International Index of Erectile Function (IIEF-5) as a diagnostic tool to study the prevalence of erectile dysfunction. Int J Impot Res, 14: 245, 2002

11. Kaplan, E. L. and Meier, P.: Nonparametric estimation from incomplete observations. J Am Stat Assoc, 53: 457, 1958

12. Boccardo, F., Rubagotti, A., Battaglia, M., Di Tonno, P., Selvaggi, F. P., Conti, G. et al: Evaluation of tamoxifen and anastrozole in the prevention of gynecomastia and breast pain induced by bicalutamide monotherapy of prostate cancer. J Clin Oncol, 23: 808,2005

13. Saltzstein, D., Sieber, P., Morris, T. and Gallo, J.: Prevention and management of bicalutamide-induced gynecomastia and breast pain: randomized endocrinologic and clinical studies with tamoxifen and anastrozole. Prostate Cancer Prostatic Dis, 8: 75, 2005

14. Tyrrell, C. J., Payne, H., Tammela, T. L., Bakke, A., Lodding, P., Goedhals, L. et al: Prophylactic breast irradiation with a single dose of electron beam radiotherapy (10 Gy) significantly reduces the incidence of bicalutamide-induced gynecomastia. Int J Radiat Oncol Biol Phys, 60: 476, 2004

15. Van Poppel, H., Tyrrell, C. J., Haustermans, K., Cangh, P. V., Keuppens, F., Colombeau, P. et al: Efficacy and tolerability of radiotherapy as treatment for bicalutamide-induced gynaecomastia and breast pain in prostate cancer. Eur Urol, 47: 587, 2005

16. Eaton, A. C., Makris, A. and Makris, A.: Once weekly tamoxifen in the prevention of gynaaecomastia and breast pain secondary to bicalutamide therapy. J Urol, suppl., 171: 282, abstract 1069,2004

17. Widmark, A., Fossa, S. D., Lundmo, P., Damber, J. E., Vaage, S., Damber, L. et al: Does prophylactic breast irradiation prevent antiandrogen-induced gynecomastia? Evaluation of 253 patients in the randomized Scandinavian trial SPCG-7/SFUO-3. Urology, 61: 145, 2003

18. Joiner, M. C.: The linear-quadratic approach to fractionation. In Basic Clinical Radiobiology. Edited by G. C. Steel. London: Edward Arnold, pp. 55-64, 1993

19. Kunkler, I.: Adjuvant irradiation for breast cancer. BMJ, 320: 1485,2000 